\title{
Computational Fluid Dynamic Analysis of Coronary Artery Stenting
}

\author{
Feng Gao, Gang Li, Rui Hu, and Hiroshi Okada
}

\begin{abstract}
Coronary artery disease is the most prevalent form of cardiovascular disease and is the largest subset of this mortality. Stent implantation becomes a common interventional procedure for treatment of coronary artery disease. In this study, fluid dynamics factors in patient specific three dimensional coronary artery models before and after stenting were studied. The three dimensional models of the coronary artery were constructed from CT image of the patient. Commercial software Adina was used for computational fluid dynamics simulation. The wall shear stress and blood velocity were greater at the region of stenosis before stenting. The fluid dynamics values have come back near to normal values in the coronary artery model fitted with stent. The technique can be employed to estimate the relevant quantities and to optimize the design of intravascular stents.
\end{abstract}

Index Terms-Computational fluid dynamics, coronary artery, wall shear stress, stenting.

\section{INTRODUCTION}

Cardiovascular disease remains the leading cause of death among women in the United States [1] and coronary artery disease is a major cause of mortality and morbidity in the Western world. Coronary artery disease is the most prevalent form of cardiovascular disease and is the largest subset of this mortality. An estimated 16.3 million Americans 20 years of age and older have Coronary artery disease, and the overall Coronary artery disease prevalence is 7 percent in adults in the United States. Ref. [1] Coronary artery disease is also called atherosclerotic heart disease in which excess cholesterol attaches itself to the walls of coronary artery vessels. Several risk factors such as smoking, hypertension, diabetes mellitus and high cholesterol have been identified which contribute to the progression of coronary artery disease. Embeded cholesterol in artery wall can also attract cellular waste products, alcium and fibrin, et al. As an atherosclerotic plaque increases in size, the insides of the coronary arteries get narrower and less blood can flow through them, usually resulting in myocardial infarction or heart attack.

Transient ischemic attack can result from thrombolytic and embolismic complications of arteriosclerosis in the coronary

Manuscript received December 25, 2013; revised March 13, 2014.

Feng Gao and Hiroshi Okada are with the Department of Mechanical Engineering, Faculty of Engineering and Technology, Tokyo University of Science, 2641 Yamazaki, Noda, Chiba, 278-8510, Japan (e-mail: gao@rs.noda.tus.ac.jp, hokada@rs.noda.tus.ac.jp).

Gang $\mathrm{Li}$ is with the Cardiac Centre of Hebei General Hospital, Shijiazhuang, Hebei 050051, China (e-mail: hrlg2002@hotmail.com).

Rui $\mathrm{Hu}$ is with Clinical Laboratory, The Second Hospital of Hebei Medical University, 100-5 Heping West Road, Xinhua District, Shijiazhuang, Hebei 050000, China (e-mail: dazuichazi2013@hotmail.com). arteries. A possible cause for these incidents is the involvement of hemodynamics, the basis for the current research activity. Hemodynamics might be defined to be an important role in the development of atherosclerotic plaques in coronary arteries. However, the specific mechanism whereby blood flow patterns influence the development of arterial disease remains a challenging topic in bioengineering. A considerable amount of evidence shows that fluid mechanics has a strong influence on the progression of several cardiovascular diseases, such as atherosclerotic and ischemic diseases [2], [3].

Stent placement becomes a common interventional procedure for treatment of coronary artery disease and its clinical outcome was better when compared to standard angioplasty [4]. Intravascular stent, which is a small tubular structure, may be driven and expanded inside passage and improve blood flow [5]. It has been applied extensively in the coronary, renal, and peripheral vascular systems. The use of intravascular stents tends to lower the complication rate. Although re-stenosis rates as high as $15-30 \%$ after six months for human coronary arteries have been observed [6]. It is difficult to perform the deployment of a stent deployed within a coronary artery and the experimental evaluation is expensive. As a result, computational numerical methods have become as powerful and indispensable method to understand the mechanical behavior of stent implantation. Recently, there has been more and more endeavor to study angioplasty and stenting procedures by means of computational structural analyses. The methodology using patient-specific artery model has the potential to provide a scientific basis for optimizing stenting treatment procedures. One possible explanation for this observation relies on the hemodynamic modifications induced by the prosthesis. Changes in wall shear stress are believed to induce endothelial dysfunction, ultimately leading to intimal hyperplasia and re-stenosis. [7] suggest that magnitude of the shear stress is of secondary importance to the spatial and temporal fluctuations of this quantity. In vivo testing performed by Vernhet et al. [8] Vernhet et al. [9] and Rolland et al.[10] show that endovascular stenting induces a large modification of the arterial compliance.

Computational cardiovascular fluid mechanics has been one of the most popular research areas in computational mechanics [11]-[13]. The geometric configuration of vascular tracts has a major role on the resulting flow patterns. Therefore, the necessity to characterize local hemodynamics inside realistic models of vasculature has been advanced. Reliable noninvasive tools for imaging the coronary arteries are being developed and enable early diagnosis and therapeutic planning. Because of advances in technology that now allow rapid image acquisition and submillimeter 
isotropic spatial resolution, CT is emerging as a potential technique for noninvasive, high-resolution evaluation of the coronary arteries [14]. Also, computed tomography (CT) and magnetic resonance (MR) allow to acquire three-dimensional (3D) anatomy with high resolution and low invasiveness. On the basis of the acquired images, image processing methods offer the possibility to virtually reconstruct $3 \mathrm{D}$ models reproducing the geometry of the vascular wall. After the definition of proper boundary conditions, the reconstructed models can then be used to perform computational simulations of local hemodynamics. This approach has recently found concrete implementations and brought to interesting results [15]-[18].

In this article we address those challenges and present a computational analysis based on patient-specific coronary artery with and without stent, where we assess the influence of the stent on the flow in the coronary artery.

\section{METHODS}

A patient with coronary artery stenosis was involved in this study. The patient was 65 years old female, presented with unstable angina. Coronary angiography revealed luminal obstruction in the middle sencond of the left anterior descending. The patient was medicated with clopidogrel 300 $\mathrm{mg}$ and acetylsalicylic acid $200 \mathrm{mg}$ on the day the angiography was carried out. On the following day, he underwent the implantation of a XIENCE V stent (Abbott Vascular, Santa Clara, California, USA) and the right femoral artery was the access via used. The patient underwent 64-slice CT-scanning both prior to the intervention and after the implantation to obtain $\mathrm{CT}$ images data.

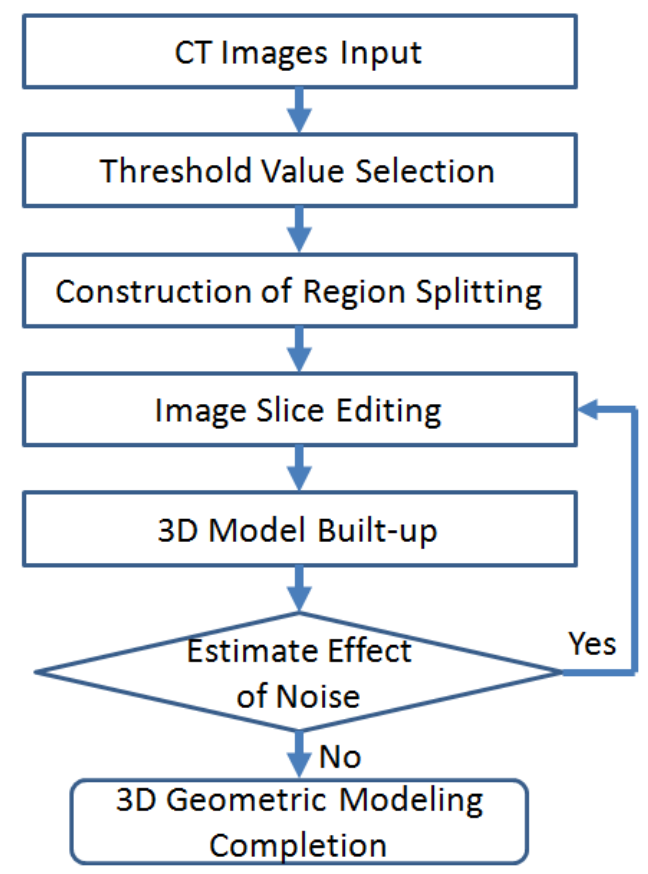

Fig. 1. Coronary geometry model construction flow chart.

The construction process of patient specific model involves the following three major steps: 1) non-invasive image acquisition, 2) imaging process, and 3) three-dimensional reconstruction to form voxel-based volumetric image representation. Figure 1 showed the flow chart regarding the construction of coronary geometry model. Mimics software (Materialise, Leuven, Belgium) is a widely-used software in the field of 3D reconstruction. Based on the parameters in the header of images file, Mimics group the data into sequence. After the raw images of coronary artery was read by Mimics, the operators determined the orientations. Mimics divides the screen into three views: the original axial view of the image, and resliced data making up the coronal and sagittal views. The built in gray threshold was used to select coronary to form different mask layers; use "image edit" feature to "add" and "erase" image boundary to increase the precision of the reconstructed image. Also, region growth feature was used to break up the image to obtain more precise coronary voxel. These data served as an input to generate a 3D geometry models of pre-interventional and post-interventional coronary artery. The realistic coronary geometry is the patient's individual coronary geometry by converting medical image data into patient-specific three-dimensional surfaces and volume representation.
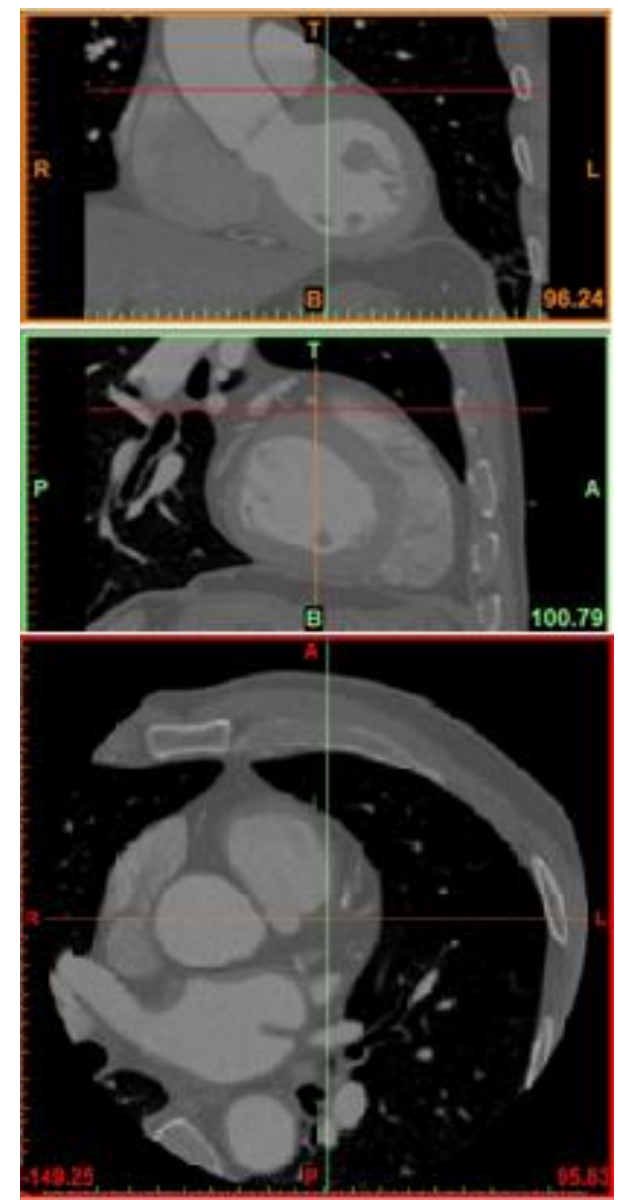

Fig. 2. The three views of coronary artery CT images in the Mimics main interface.

STL files for pre- and post-stenting models were imported into ADINA 8.7 (ADINA R \& D, Inc., USA). ADINA creates the necessary points, body edges and faces that can be used to define finite element meshes and also loads and boundary conditions. The pre- and post-stenting models were generated by tetrahedral volume meshes using ADINA-F. Fig. 3 shows the model for coronary artery before and after stenting. 


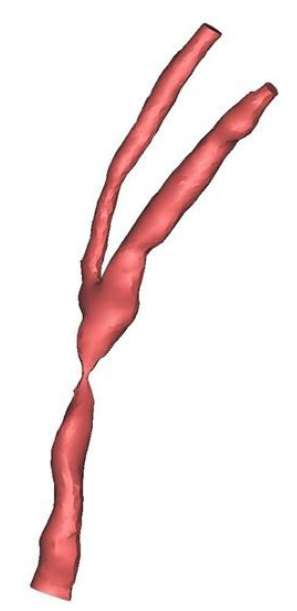

Before stenting

After stenting

The flow is assumed to be laminar Newtonian, viscous and incompressible. The blood can be represented by an incompressible fluid which is governed by the Navier-Stokes equations

$$
p v \times \nabla v=-\nabla \times \tau-\nabla p
$$

and the continuity equaiton

$$
\nabla \times v=0
$$

where $v$ is the $3 \mathrm{D}$ velocity vector, $P$ is the pressure, $\rho$ is the density and $\tau$ is the shear stress term. Average physiological hemodynamic conditions with steady flow have been considered for the 3D numerical simulations. The blood was assumed to behave as a Newtonian fluid. The viscosity was set to $0.0035 \mathrm{~Pa} . \mathrm{s}$ and the density to $1060 \mathrm{~kg} / \mathrm{m} 3$, corespongding to the standard values cited in the literature [19]. The Navier-Stokes equations provide a differential expression of the mass and momentum conservation laws. The reference value $0.2 \mathrm{~m} / \mathrm{s}$ [20] of the flow velocity was applied at the inlet for both models. A zero traction force outlet boundary condition was applied to both outlets. The ADINA 8.7 is used to numerically simulate the blood flow in pre- and post-stenting coronary models.
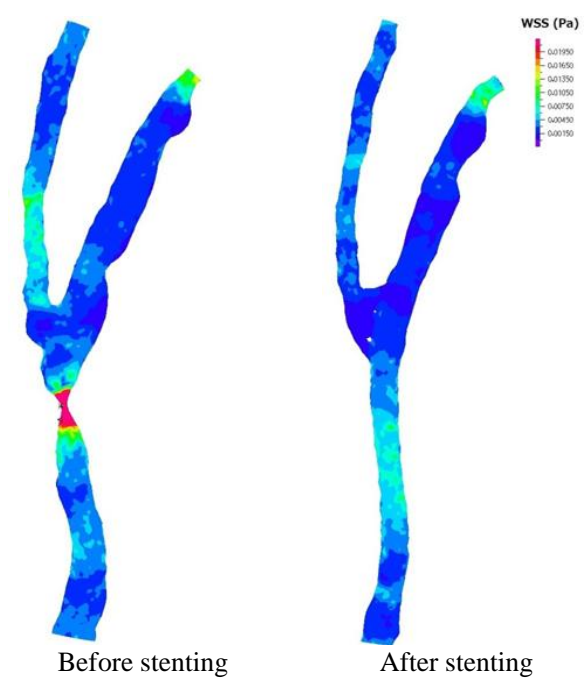

Fig. 4. Wall shear stress in coronary artery bifurcation before and after stenting.

\section{RESULTS AND DISCUSSION}

Patient-specific analysis of the flow in coronary artery with a stent also involves computational challenges, mostly related to the need to have a good spatial representation of the stent. There are only a few studies that have utilized patient-specific derived arterial geometries in the analysis of stent [21]-[23]. The methodology using patient-specific artery model has the potential to provide a scientific basis for optimizing stenting treatment procedures.

The velocity is mall for the flow at the artery's inlet. It is observed that there is an increase in velocity at the stenosis before stenting, but at the branches the flow behavior appears to be normal. In the presence of a stenosis, a sharp velocity profile is formed. A jet-like flow emerges from the constricted region severely affecting the downstream flow field. At the inlet the flow velocity is $0.2 \mathrm{~m} / \mathrm{s}$, but the maximum flow rate of blood flow at stenosis is $4.0 \mathrm{~m} / \mathrm{s}$. On the contrary, after the stent implantation, the flow recovers to normal profile and the overall distribution remains nearly the same. After the stent implantation of the coronary artery, and at the same inlet velocity of $0.2 \mathrm{~m} / \mathrm{s}$, the maximum flow rate of blood flow in coronary artery decreased to $0.5 \mathrm{~m} / \mathrm{s}$. After stenting, the jet flow pattern in the stenosis recovered to blunted flow. The angiographic severity of coronary stenoses correlates with impaired coronary flow velocity reserve [24], [25]. An impaired coronary flow velocity reserve immediately after optimal stent implantation has been reported to be caused by an elevated basal average peak velocity rather than an attenuated augmentation of the average peak velocity [26], [27]. A lumen enlargement of lesions with stents, the impaired coronary flow velocity reserve may normalize in weeks or months following the procedure [27].

There is a region of high pressure in pre-stenting coronary artery model and it is situated before the stenosis. For the coronary artery model after stent implantation, the high pressure was reduced. In stenosis coronary artery, blood was accumulated before the plaque region. Hence an increase of blood pressure was observed before the plaque region in the coronary artery. After stent implantation, blood pressure pattern was maintained in the normal range by the fitted stent at stenosis region. In the present study, the inlet velocity is set to be the same for both the models. After stent implantation, blood flow resistance decreases in the vessel. Therefore, the wall pressure was decreased.

Distribution of wall shear stress in coronary artery bifurcation before and after stenting are shown in Figure 6 . Large wall shear stress exists at the stenosis region and the highest wall shear stress corresponded to the most severely stenotic segment. The maximum wall shear stress of the stenotic portion of the coronary artery was $0.13 \mathrm{~Pa}$. After stent implantation, the value of wall shear stress in coronary artery bifurcation was reduced. As shown in Figure 4, there is no region where the value of wall shear stress is larger than $0.02 \mathrm{~Pa}$ in coronary artery bifurcation model. These results indicate that stent has greater effect of reducing wall shear stress at stenotic coronary artery. $\mathrm{Ku}$ et al. [28] found significant positive correlation between low and oscillating shear stress and wall thickness by measuring velocity using laser Doppler anemometry in a averaged Plexiglas model of 
the carotid bifurcation and intimal thickness on histological sections. With the development of numerical methods and the availability of computational resources experienced in the last decade, the experimental approach has been progressively replaced by the computational approach, even if it still represents a fundamental step for computational model validation [29], [30]. In general, computational fluid dynamics methods allow to indicate flow patterns, particle residence times and shear stress distributions at a great level of detail and to easily alter simulation parameters. Increasingly complex models of arterial segments have been generated and studied over the years [31]-[33], [11]-[13], leading to a deeper understanding of the influence of geometry, flow waveforms, viscosity and wall distensibility on flow and shear stress patterns.

\section{REFERENCES}

[1] V. L. Roger, A. S. Go, D. M. Lloyd-Jones et al., "Heart Disease and Stroke Statistics-2012 Update: A Report from the American Heart Association," Circulation, vol. 125, no. 1, pp. e2-e220, 2012.

[2] D. N. Ku, "Blood Flow in Arteries," Annu. Rev. Fluid Mech., vol. 29, pp. 399-434, 1997.

[3] S. A. Berger and L. D. Jou, "Flow in Stenotic vessels," Апnи. Rev. Fluid Mech., vol. 32, pp. 347-382, 2000.

[4] P. W. Serruys, P. de Jaegere, and F. Kiemeneij, "A comparison of balloon-expandable-stent implantation with balloon angioplasty in patients with coronary artery disease," $N$ Eng. J. Med., vol. 331, pp.489-495, 1994.

[5] C. T. Dotter, "Transluminally-placed coil spring end arterial tube grafts, long-term patency in canine popliteal artery," Investigative Radiology, vol. 4, pp. 329-332, 1969.

[6] T. Rau, J. Schofer, M. Schluter, A. Seidensticker, J. Berger, and D. G. Mathey, "Stenting of non acute total coronary occlusions: predictors of late angiographic outcome," J. Am. Coll. Card., vol. 31, pp. 275-280, 1998.

[7] P. Davies, C. Shi, N. DePaola, B. Helmke, and D. Polacek, "Hemodynamics and the focal origin of atherosclerosis. A spatial approach to endothelial structure, gene expression, and function," Annu. N.Y. Acad. Sci., vol. 947, pp. 7-16, 2001.

[8] H. Vernhet, R. Demaria, M. C. Oliva-Lauraire, J. Juan, J. P. Senac, and M. Dauzat, "Changes in wall mechanics after endovascular stenting in rabbit aorta: comparison of three diffierent stent designs," Am. J. Roent, vol. 176, pp. 803-807, 2001.

[9] H. Vernhet, J. M. Juan, R. Demaria, M. C. Oliva-Lauraire, J. P. Senac, and M. Dauzat, "Acute changes in aortic wall mechanical properties after stent placement in rabbits," J. Vasc. Inter. Rad., vol. 11, pp. 634-638, 2000.

[10] P. H. Rolland, A. B. Charifi, and C. Verrier, "Hemodynamics and wall mechanics after stent placement in swine illiac arteries: comparative results from six stent designs," Radiology, vol. 213, pp. 229-246, 1999.

[11] R. Torii, M. Oshima, T. Kobayashi, K. Takagi, and T. E. Tezduyar, "Influence of wall elasticity in patient-specific hemodynamic simulations," Comput. Fluids, vol. 36, pp. 160-168, 2007.

[12] K. Takizawa, K. Schjodt, A. Puntel, N. Kostov, and T. E. Tezduyar, "Patient-specific computer modeling of blood flow in cerebral arteries with aneurysm and stent," Comput Mech., vol. 50, no. 6, pp. 675-686, 2012.

[13] W. Fu, Z. Gu, X. Meng, B. Chu, and A. Qiao, "Numerical Simulation of Hemodynamics in Stented Internal Carotid Aneurysm Based on Patient-Specific Model," Journal of Biomechanics, vol. 43, no. 7, pp. 1337-1342, 2010.

[14] A. K. Attili and P. N. Cascade, "CT and MRI of coronary artery disease: evidence-based review," AJR Am J Roentgenol., vol. 187, suppl. 6, pp. S483- S499, 2006.

[15] B. Ene-Iordache, L. Mosconi, G. Remuzzi, and A. Remuzzi, "Computational fluid dynamics of a vascular access case for hemodyalsis," Journal of Biomechanical Engineering, vol. 123, no. 3, pp. 284-292, June 2001.

[16] G. van Langenhove, J. J. Wentzel, R. Krams, C. J. Slager, J. N. Hamburger, and P. W. Serruys, "Helical velocity patterns in a human coronary artery. A three-dimensional computational fluid dynamic reconstruction showing the relation with local wall thickness," Circulation, vol. 102, pp. e22-e24, 2000.
[17] K. Perktold, M. Hofer, G. Rappitsch, M. Loew, B. D. Kuban, and M. H Friedman. "Validated computation of physiologic flow in a realistic coronary artery branch," Journal of Biomechanics, vol. 31, pp. 217-228, 1998.

[18] D. A. Steinman, "Image-based CFD modeling in realistic arterial geometries," Annals of Biomedical Engineering, vol. 30, no. 4, pp. 483-497, 2002.

[19] S. A. Berger, W. Goldsmith, and E. R. Lewis, Introduction to Bioengineering, New York, NY: Oxford University Press, 1996

[20] B. M. Johnston, P. R. Johnston, S. Corney, and D. Kilpatrick, "Non-Newtonian blood flow in human right coronary arteries: steady state simulations," J. Biomech vol. 37, no. 5, pp. 709-720, 2004.

[21] G. Holzapfel, M. Stadler, and C. Schulze-Bauer, "A layer-specific three-dimensional model for the simulation of balloon angioplasty using magnetic resonance imaging and mechanical testing," Ann Biomed Eng., vol. 30, pp. 753-767, 2002

[22] D. Kiousis, T. Gasser, and G. Holzapfel, "A numerical model to study the interaction of vascular stents with human atherosclerotic lesions," Ann Biomed Eng., vol. 35, pp. 1857-1869, 2007.

[23] F. Gijsen, F. Migliavacca, and S. Schievano, "Simulation of stent deployment in a realistic human coronary artery," BioMed Eng OnLine, vol. 7, no. 23, 2008

[24] K. L. Gould, K. Lipscomb, and G. W. Hamilton, "Physiologic basis for assessing critical coronary stenosis. Instantaneous flow response and regional distribution during coronary hyperemia as measures of coronary flow reserve," Am J Cardiol, vol. 33, pp. 87-94, 1974.

[25] J. J. Piek, E. Boersma, and C. di Mario, "Angiographical and Doppler flow-derived parameters for assessment of coronary lesion severity and its relation to the result of exercise electrocardiography. DEBATE study group. Doppler Endpoints Balloon Angioplasty Trial Europe," Eur Heart J., vol. 21, pp. 466-74, 2000.

[26] K. J. Kern, S. Puri, and R. G. Bach, "Abnormal coronary flow velocity reserve after coronary artery stenting in patients: role of relative coronary reserve to assess potential mechanisms," Circulation, vol 100, pp. 2491-2498, 1999.

[27] R. A. van Liebergen, J. J. Piek, K. T. Koch, R. J. de Winter, and K. I. Lie, "Immediate and long-term effect of balloon angioplasty or stent implantation on the absolute and relative coronary blood flow velocity reserve," Circulation, vol. 98, pp. 2133-2140, 1998.

[28] D. N. Ku, D. P. Giddens, C. K. Zarins, and S. Glagov, "Pulsatile flow and atherosclerosis in the human carotid bifurcation positive correlation between plaque location and low oscillating shear stress," Arteriosclerosis, vol. 5, pp. 293-302, 1985.

[29] R. Botnar, G. Rappitsch, M. B. Scheidegger, D. Liepsch, K. Perktold, and P. Boesiger, "Hemodynamics in the carotid artery bifurcation: a comparison between numerical simulations and in vitro MRI measurements," Journal of Biomechanics, vol. 33, pp. 137-144, 2000

[30] K. Perktold, M. Hofer, G. Rappitsch, M. Loew, B. D. Kuban, and M. H Friedman, "Validated computation of physiologic flow in a realistic coronary artery branch," Journal of Biomechanics, vol. 31, pp. 217-228, 1998.

[31] C. R. Ethier, D. A. Steinman, X. Zhang, S. R. Karpik, and M. Ojha, "Flow waveform effects on end-to-side anastomotic flow patterns," Journal of Biomechanics, vol. 31, pp. 609-617, 1998.

[32] P. E. Hughes and T. V. How, "Flow structures at the proximal side-to-end anastomosis. influence of geometry and flow division," Transactions of the ASME, vol. 117, pp. 224-236, 1995.

[33] S. Hyun, C. Kleinstreuer, and J. P. Archie, "Hemodynamics analyses of arterial expansions with implications to thrombosis and restenosis," Medical Engineering and Physics, vol. 22, pp. 13-27, 2000.

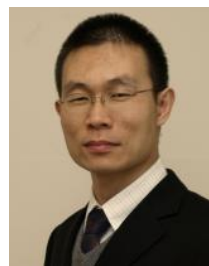

Feng Gao was born in Shandong, China in 1977. He received B.S. degree in mechanical engineering from Nanjing Forest University, Jiangsu, China, in 2000, M.S. degree in mechanics from Beijing University of Technology, Beijing, China in 2003, and Ph.D. degree in information science in Japan Advanced Institute of Science and Technology, Ishikawa, Japan, in 2006.

$\mathrm{He}$ continued his postdoctoral training in Aalborg Hospital Science and Innovation Center, Aalborg, Denmark until 2008. He was a research scientist in Department of Bioengineering at University of Washington, Seattle, USA, from 2008 to 2010. Currently, he is an assistant professor in Department of Mechanical Engineering at Tokyo University of Science, Chiba, Japan. His research interests include biomechanics, computational modeling and numerical simulation in physiology, and fluid-structure interaction

Dr. Gao is a member of Japan Society for Computational Engineering and Science. 


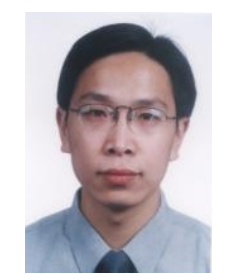

Gang Li was born in Liaoning, China in 1977. He received M.D. degree from Hebei Medical University, Hebei, China, in 2001, and Ph.D. degree from Shinshu University Graduate School of Medicine, Matsumoto, Japan, in 2007.

He continued his postdoctoral training in Department of Metabolic Regulation, Shinshu University, Matsumoto, Japan until 2008. Currently, he is an

associate professor in Cardiovascular Center of Hebei General hospital, Hebei ,China. His research interests and clinical activities mainly dealt with prevention of complications after cardiac interventional therapy.

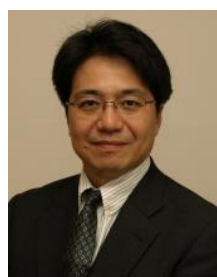

Hiroshi Okada was born in Kagoshima, Japan. He received B.S. degree in mechanical engineering from Tokyo University of Science, Chiba, Japan, in 1986 , and Ph.D. degree in mechanical engineering in Division of Engineering School of Civil Engineering at Georgia Institute of Technology, Atlanta, USA in 1990.

He continued his postdoctoral training in Georgia Institute of Technology, Atlanta, USA until 1991. He then joined Nissan Research Center, Japan, as a researcher. From 1993 to 1996, he was appointed as a research scientist in Computational Mechanics Center, Georgia Institute of Technology, Atlanta, USA, and an ASSOCIATE PROFESSOR at Kagoshima University, Japan. Currently, he a PROFESSOR in Department of Mechanical Engineering, Faculty of Science and Technology at Tokyo University of Science, Chiba, Japan. He is specialized in Machine material/material mechanics, Finite Element Method, Fracture Mechanics, and Simulation.

Prof. Okada has served, and is serving, as a member of numerous technical committees of professional organizations such as Japan Society of Mechanical Engineers (JSME), Japan Society for Computational Engineering and Science (JSCES), Japan Association for Computational Mechanics (JACM), Japan Welding Engineering Society (JWES), and as editor for Journal of Computational Science and Technology, and as a scientific committee chair or as chair of programs, for conference of WCCM, JSME, JSCES. He was awarded The K. Washizu Medal in 2008, Computational Mechanics Achievement Award from JSME and JACM Fellows Award in 2009. 